\title{
Do neurologists agree in diagnosing drug resistance in adults with focal epilepsy?
}

\author{
Gaetano Zaccara' | Marco Mula ${ }^{2}$ (D) | Bruno Ferrò ${ }^{3}$ | Domenico Consoli ${ }^{4}$ (iD | \\ Maurizio Elia ${ }^{5}$ (D) | Anna Teresa Giallonardo ${ }^{6}$ | Alfonso Iudice ${ }^{7}$ | Angela La Neve ${ }^{8}$ | \\ Stefano Meletti ${ }^{9}$ (D) | Paolo Tinuper ${ }^{10,11}$ | Leila Zummo ${ }^{12}$ Emilio Perucca ${ }^{13}$
}

${ }^{1}$ Regional Health Agency of Tuscany, Florence, Italy

${ }^{2}$ Atkinson Morley Regional Neuroscience Centre, St George's University Hospitals NHS Foundation Trust and Institute of Medical and Biomedical Education, St George's University of London, London, UK

${ }^{3}$ UCB Pharma SpA, Milan, Italy

${ }^{4}$ Vibo Valentia Health Authority, Vibo

Valentia, Italy

${ }^{5}$ Oasi Research Institute-IRCCS, Troina, Italy

${ }^{6}$ Department of Neurology and Psychiatry, "Sapienza" University, Rome, Italy

${ }^{7}$ Department of Clinical and Experimental Medicine, Section of Neurology, University of Pisa, Pisa, Italy

${ }^{8}$ Department of Basic Medical Sciences, Neurosciences and Sense Organs, University of Bari, Bari, Italy

${ }^{9}$ Department of Biomedical, Metabolic, and Neural Science, University of Modena and Reggio Emilia, Modena, Italy

${ }^{10}$ IRCCS Institute of Neurological Sciences of Bologna, Bologna, Italy

${ }^{11}$ Department of Biomedical and Neuromotor Sciences, University of Bologna, Bologna, Italy

${ }^{12}$ Department of Experimental Biomedicine and Clinical Neurosciences (BIONEC), University of Palermo, Palermo, Italy

\section{Summary}

Objective: To evaluate interrater agreement in categorizing treatment outcomes and drug responsiveness status according to the International League Against Epilepsy (ILAE) definition of drug-resistant epilepsy.

Methods: A total of 1053 adults with focal epilepsy considered by the investigators to meet ILAE criteria for drug resistance were enrolled consecutively at 43 centers and followed up prospectively for 18-34 months. Treatment outcomes for all antiepileptic drugs (AEDs) used up to enrollment (retrospective assessment), and on an AED newly introduced at enrollment, were categorized by individual investigators and by 2 rotating members of a 16-member expert panel (EP) that reviewed the patient records independently. Interrater agreement was tested by Cohen's kappa $(k)$ statistics and rated according to Landis and Koch's criteria.

Results: Agreement between EP members in categorizing outcomes on the newly introduced AED was almost perfect $(90.1 \%, k=0.84$, 95\% confidence interval [CI] 0.80-0.87), whereas agreement between the EP and individual investigators was moderate $(70.4 \%, k=0.57,95 \%$ CI $0.53-0.61)$. Similarly, categorization of outcomes on previously used AEDs was almost perfect between EP members $(91.7 \%, k=0.83,95 \%$ CI 0.81-0.84) and moderate between the EP and investigators $(68.2 \%, k=0.50,95 \%$ CI $0.48-0.52)$. Disagreement was related predominantly to outcomes considered to be treatment failures by the investigators but categorized as undetermined by the EP. Overall, $19 \%$ of patients classified as having drug-resistant epilepsy by the investigators were considered by the EP to have "undefined responsiveness."

Significance: Interrater agreement in categorizing treatment outcomes according to ILAE criteria ranges from moderate to almost perfect. Nearly 1 in 5 patients considered by enrolling neurologists to be "drug-resistant" were classified by the EP as having "undefined responsiveness." 
${ }^{13}$ Clinical Pharmacology Unit, Department of Internal Medicine and Therapeutics, University of Pavia and Clinical Trial Center, IRCCS Mondino Foundation, Pavia, Italy

\section{Correspondence}

Gaetano Zaccara, Regional Health Agency of Tuscany, Florence, Italy.

Email: gaetanozaccara@yahoo.it

\section{K E Y W O R D S}

antiepileptic drugs, classification, drug-resistant epilepsy, epilepsy, ILAE definition, reliability

\section{1 | INTRODUCTION}

Although there is general agreement that drug resistance is a major challenge in the management of epilepsy, definitions of pharmacoresistance used in past decades have differed widely across studies. ${ }^{1,2}$ To promote the use of common concepts and a common language, in 2009 the International League Against Epilepsy (ILAE) Task Force proposed to define drug resistance as "failure of adequate trials of two tolerated and appropriately chosen and used antiepileptic drug (AED) schedules (whether as monotherapies or in combination) to achieve sustained seizure freedom." 3 The assessment of the possible outcomes of an intervention (seizure-free, treatment failure, and undetermined) by ILAE criteria is largely conceptual, ${ }^{4}$ and the categorization process needs to be operationalized to determine when a therapeutic trial is "appropriate" (selection of AED based on seizure and patient characteristics) and "adequate" (including good tolerance, optimal dosing schedules, drug adherence and duration of exposure). In situations where appropriateness and adequacy cannot be assessed or satisfied, the outcome is deemed as "undetermined." The ILAE categorization scheme is increasingly being applied worldwide in studies on drug-resistant epilepsy. ${ }^{6,7}$

The ILAE Task Force emphasized that the proposed definition should be regarded as a "working framework" and that prospective validation studies should be encouraged. Based on these premises, a large prospective study was initiated in 2011 with the following 2 main objectives: (a) to assess outcomes on a newly introduced AED treatment in adults meeting ILAE criteria for drug-resistant focal epilepsy and (b) to test interrater variability among neurologists in applying ILAE criteria for categorizing treatment outcomes and drug resistance status. To investigate the reliability of the ILAE definition, this study involved a comparison of the categorization of outcomes made by individual neurologists with that made by an external expert panel (EP), as well as a comparison of level of agreement between EP members. The results of this comparison represent the focus of this article.

\section{Key Points}

- Interrater agreement in categorizing treatment outcomes by ILAE criteria is moderate to almost perfect, being dependent on the expertise of individual raters

- Based on an EP's assessment, 1 in 5 patients considered to be drug resistant by the treating neurologists actually have undefined responsiveness

- There is a need to have a better understanding of the ILAE definition of drug resistance, and to take a more critical approach to evaluating treatment outcomes

\section{2 | METHODS}

\section{1 | Study design, eligibility criteria, and study procedures}

Data were drawn from a large multicenter, observational, prospective study of adults with drug-resistant focal epilepsy recruited in 43 epilepsy centers in Italy (Appendix) between 2011 and 2015, aimed at investigating treatment outcomes after introduction of another (additional or substituted) AED (Prometeo study SP0992). ${ }^{8}$ Patients were enrolled consecutively according to the following criteria: age 18 years or older; an established diagnosis of focal epilepsy; a diagnosis of drug resistance made by the enrolling physician according to ILAE criteria ${ }^{3}$; an interseizure interval not exceeding 6 months in the 12 months preceding enrollment; being on AED treatment with a clinical indication to modify existing therapy by introducing another AED, either as add-on or in substitution, in a combination not previously used.

Prior to initiation of enrollment, investigators participated in an ad hoc interactive meeting to ensure that all were familiar with the ILAE definition of drug resistance and with the variables needed to categorize patients. It was emphasized that, in accordance with the observational nature of the study, treatments had to be prescribed according 
to good medical practice and that, in particular, adequacy of dosing schedules had to be assessed based on clinical judgement, using the World Health Organization (WHO) defined daily dose as a rough reference only. ${ }^{3}$ To determine whether potentially eligible patients fulfilled ILAE criteria for drug resistance, investigators assessed outcomes of all previously administered treatments based on clinical records and an interview with the patient. Dosages of the newly introduced AED and other medications were adjusted as clinically indicated, and the outcome of the treatment introduced at enrollment was evaluated prospectively according to ILAE criteria. Patients were assessed at time 0 (baseline) and at 6, 12 , and 18 months, or more frequently if clinically indicated. An additional visit was scheduled for patients who had been seizure-free for at least 60 days at the 18-month visit but had not yet met ILAE criteria for seizure freedom. In these patients, follow-up could be extended for up to an additional 16 months to provide sufficient time to establish whether seizure freedom had been achieved.

The study was conducted in compliance with Good Clinical Practice guidelines ICH. Approval was obtained from ethics committees of all participating centers, and written informed consent was obtained from each patient. Information recorded in the electronic case report forms (eCRFs) included demographic and disease-related characteristics, outcome data (reported in a separate publication ${ }^{8}$ ), and treatment details (dosing schedules, titration rates, and serum drug concentrations, when available) for each AED and other drugs used by the patient in the past and throughout the study.

\subsection{Role of the expert panel (EP)}

An expert panel (EP) composed of 16 experienced epileptologists, not involved in the enrollment or management of study patients or in the compilation of eCRFs at local sites, reviewed all eCRFs to confirm or refute the investigators' categorization of the outcome of each AED trial applied before enrollment (retrospective categorization) and of the AED newly introduced at enrollment (prospective categorization), as well as the categorization of each patient's drug responsiveness/resistance status. Before initiation of the reviewing process, each EP member categorized treatment outcomes in a sample of $30 \mathrm{eCRF}$ obtained from a separate set of patients, and the results of this review were compared in a meeting of all EP members in order to discuss the correct criteria of categorization.

Each eCRF was reviewed separately and independently by 2 EP members chosen in a random and blinded manner by an automated computerized system. In no case did the system assign an eCRF to an expert from an institution at which that patient was enrolled. The computerized system selected a fresh expert pair at each assignment, and each expert was assigned
eCRFs from different centers. Experts performed their evaluations without knowledge of the identity of the other expert included in the review of the same eCRF, but in case of disagreement, the computer informed the members of the EP pair that a discrepancy had occurred. At that point, the evaluation of that eCRF was unblinded and the EP pair was requested to communicate and resolve the disagreement. If the disagreement was not resolved, the chair of the EP reviewed the case and made a final categorization. Because the overall framework of the ILAE definition is comprised of 2 "hierarchical" levels, that is, determination of outcome of each AED trial (level 1) and determination of drug resistance/responsiveness status (level 2 ), both categorization levels were reviewed. Wherever the members of the EP disagreed with the investigator's categorization, the reason for disagreement had to be specified.

\section{3 | Statistical analysis}

Interrater agreement in categorizing the outcome of each drug trial (level 1) was evaluated between experts, both before and after communication, as well as between the EP and investigators. This analysis was performed separately for retrospective data (all treatments used in the past, including those present at the time of enrolment) and prospective data (outcome of the treatment introduced at enrollment). Analysis was focused on the 3 main outcomes (seizure freedom, treatment failure, undetermined). Interrater agreement on the presence/absence of adverse effects (an additional component of the ILAE categorization) was virtually negligible and was not analyzed.

Descriptive statistics (means, frequencies, and proportions) were used to characterize demographic and clinical variables. The Cohen kappa $(k)$ statistical method was used to assess interrater agreement and was interpreted according to the criteria of Landis and Koch, ${ }^{9}$ as follows: $\leq 0$, poor agreement; 0.01-0.2, slight agreement; 0.21-0.4, fair agreement; 0.41-0.6, moderate agreement; 0.61-0.8, substantial agreement; and 0.81-1, almost perfect agreement.

\section{3 | RESULTS}

\subsection{Characteristics of the study population}

The main characteristics of the study population are shown in Table 1. Each of the 1053 recruited patients had a trial on a newly introduced AED, which was prospectively evaluated. Patients had a median seizure frequency of 5 seizures per month prior to entry into the study and were taking an average of 2.1 AEDs at enrollment. The total number of AED trials evaluated at enrollment (previous and ongoing AEDs at enrolment) was 5725. One hundred seventy-two trials (3\%) were not evaluated and were excluded from the current analysis. These trials were made 
TABLE 1 Clinical characteristics of the patients enrolled in the study

\begin{tabular}{|l|l|}
\hline Age $(\mathrm{y})$ & $44.5 \pm 14.2$ \\
\hline Gender, male/female & $504 / 549$ \\
\hline $\begin{array}{l}\text { Age at epilepsy onset }(\mathrm{y}) \\
\text { Duration of the epilepsy (y) }\end{array}$ & $18.1 \pm 9.7$ \\
\hline $\begin{array}{l}\text { Seizure frequency (per } 28 \text { days) in the } 6 \text { months prior to enrollment } \\
\text { (median and range) }\end{array}$ & $5(0.1-220)$ \\
\hline All seizures ( $\mathrm{n}=1053)$ & $4(0.1-180)$ \\
\hline $\begin{array}{l}\text { Focal aware seizures }(\mathrm{n}=359) \\
\text { Focal impaired awareness seizures ( } \mathrm{n}=765)\end{array}$ & $4(0.1-180)$ \\
\hline $\begin{array}{l}\text { Focal to bilateral tonic-clonic seizures }(\mathrm{n}=326) \\
\text { Number of AEDs taken at enrollment }\end{array}$ & $1(0.1-45)$ \\
\hline $\begin{array}{l}\text { Number of previously tried AEDs (including those } \\
\text { taken at enrollment) }\end{array}$ & $2.1 \pm 0.8$ \\
\hline
\end{tabular}

$\mathrm{n}=1053$.

Results are means \pm standard deviation (SD) unless stated otherwise.

with fixed-dose combination AED products or with obsolete active principles that are no longer available.

\section{2 | Interrater agreement in categorizing outcomes on the newly introduced drug (level 1)}

The level of agreement between members of the EP and between the EP and investigators in categorizing treatment outcomes on the newly introduced AED (prospective assessment) is summarized in Table 2. The agreement between EP members after initial blind evaluation was almost perfect, with concordant categorizations being reported in 954 of 1053 cases $(90.1 \%, k=0.84$, 95\% confidence interval [CI] 0.80-0.87), and improving further after EP members communicated to solve discrepancies $(96.3 \%$, $k=0.94,95 \%$ CI $0.92-0.96)$. The magnitude of the agreement between the EP and individual investigators was moderate $(70.4 \%, k=0.57,95 \%$ CI $0.53-0.61)$, with EP and investigators failing to agree in 312 of 1053 cases
(29.6\%). In the majority of cases, outcomes that were considered by investigators as "treatment failures" (242/642, $38 \%)$ or as "seizure freedom" $(14 / 92,15 \%)$ were classified by the EP as "undetermined." Conversely, of 315 outcomes categorized as "undetermined" by investigators, 55 (17\%) were recategorized by the EP as "treatment failures" and 3 (1\%) were classified as "seizure-free."

With respect to the most common causes of disagreement, in almost two-thirds of the cases, members of the EP panel considered the maximum AED dose attained as insufficient to justify a categorization as "treatment failure" (Table 3). Inadequate dosing was also the most frequent cause of disagreement between members of the EP, both at initial assessment and after communication.

Overall, treatment outcome on the newly introduced AED was categorized as a "treatment failure" in 646 cases $(61.3 \%)$ by the investigators, and in 452 cases (43\%) by the EP. Outcome was classified as "undetermined" in 315 cases $(29.9 \%)$ according to the investigators, and in 517 cases $(49.1 \%$ ) according to the EP. Details of treatment outcomes on the newly introduced AED and associated variables are reported in a separate publication. ${ }^{8}$

The agreement between members of the EP and between the EP and investigators in categorizing treatment outcomes was also evaluated for the 5725 retrospective AED trials (eg, trials with previously used AEDs and with AEDs already in use at enrollment; Table 4). Similar to the findings of the prospective assessment, while the agreement between EP members at initial evaluation was almost perfect $(91.7 \%, k=0.83,95 \%$ CI $0.81-0.84)$ and improved further after communication between experts $(95.9 \%$, $k=0.91,95 \%$ CI $0.90-0.92$ ), the categorization of the EP differed from that of the investigator in $31.8 \%$ of cases, which corresponds to a moderate level of agreement ( $k=0.50$, 95\% CI 0.48-0.52). The most common disagreement consisted in the EP recategorizing outcomes that had been considered "treatment failures" by the investigator (1689/4786, 35.3\%) as "undetermined." In a small number of cases, outcomes were reclassified from "seizure-free" to

\begin{tabular}{|c|c|c|c|c|c|}
\hline & $\begin{array}{l}\text { Seizure } \\
\text { freedom }\end{array}$ & $\begin{array}{l}\text { Treatment } \\
\text { failure }\end{array}$ & Undetermined & $\begin{array}{l}\text { No } \\
\text { agreement }\end{array}$ & $\begin{array}{l}\text { Cohen's kappa } \\
\text { coefficient } \\
(95 \% \text { CI })\end{array}$ \\
\hline $\begin{array}{l}\text { Agreement between } \\
\text { investigators and EP }\end{array}$ & 78 & 402 & 261 & 312 & $0.57(0.53-0.61)$ \\
\hline $\begin{array}{l}\text { Agreement between } \\
\text { experts at initial } \\
\text { evaluation }\end{array}$ & 84 & 425 & 445 & 99 & $0.84(0.80-0.87)$ \\
\hline $\begin{array}{l}\text { Agreement between } \\
\text { experts after attempt } \\
\text { to resolve } \\
\text { discrepancies }\end{array}$ & 89 & 444 & 481 & 39 & $0.94(0.92-0.96)$ \\
\hline
\end{tabular}

TABLE 2 Level of agreement between investigators and $\mathrm{EP}$, and between EP members, in categorizing outcomes on a newly introduced AED (prospective assessment) in the 1053 patients included in the study 
TABLE 3 Main reasons for disagreement in categorizing the outcome of the therapeutic intervention applied at enrollment in the 1053 patients included in the study

\begin{tabular}{|c|c|c|c|}
\hline $\begin{array}{l}\text { Cause for } \\
\text { failing to } \\
\text { agree }\end{array}$ & $\begin{array}{l}\text { Disagreement } \\
\text { between } \\
\text { investigators } \\
\text { and each of } \\
\text { the } 2 \text { EP } \\
\text { members } \\
(n=632)\end{array}$ & $\begin{array}{l}\text { Disagreement } \\
\text { between EP } \\
\text { members at } \\
\text { initial } \\
\text { evaluation } \\
(n=279)\end{array}$ & $\begin{array}{l}\text { Disagreement } \\
\text { between EP } \\
\text { members } \\
\text { after attempt } \\
\text { to resolve } \\
(n=87)\end{array}$ \\
\hline $\begin{array}{l}\text { Lack of } \\
\text { adequate } \\
\text { information }\end{array}$ & $56(8.9 \%)$ & $23(8 \%)$ & $10(11 \%)$ \\
\hline $\begin{array}{l}\text { Inappropriate } \\
\text { AED choice }\end{array}$ & $4(0.6 \%)$ & $4(1.4 \%)$ & 0 \\
\hline $\begin{array}{l}\text { Insufficient } \\
\text { seizure- } \\
\text { freedom } \\
\text { period }\end{array}$ & $9(1.4 \%)$ & $2(0.7 \%)$ & $5(6 \%)$ \\
\hline $\begin{array}{l}\text { Insufficient } \\
\text { treatment } \\
\text { duration }\end{array}$ & $6(0.9 \%)$ & $2(0.7 \%)$ & - \\
\hline $\begin{array}{l}\text { Insufficient } \\
\text { AED dose }\end{array}$ & $396(63 \%)$ & $173(62 \%)$ & $57(65 \%)$ \\
\hline $\begin{array}{l}\text { Other (not } \\
\text { specified) }\end{array}$ & $161(25 \%)$ & 75 (27\%) & $15(17 \%)$ \\
\hline
\end{tabular}

$n$, total number of reasons for which there was disagreement. Each of the 2 members of the EP may have expressed a different reason for disagreement and therefore the number of reasons may exceed the number of disagreements in the categorization of outcomes.

"undetermined" (19/77, 24.7\%) or from "undetermined" to "treatment failure" (107/862, 12.4\%). As reported for the prospective assessment, use of AED doses considered to be adequate by the investigator and to be insufficient by the EP was the most common reason for disagreement (46\% of cases) and was followed by lack of adequate information ( $41 \%$ of cases). The latter was also the most common cause of disagreement between EP members.

\subsection{Interrater agreement in categorizing drug-resistance status (level 2)}

Figure 1 illustrates the distribution of the number of previous AED failures for the 1053 patients at the time of enrollment, as categorized by the investigators and by the EP, respectively. All patients were categorized by the investigators as being drug resistant, even though in 4 cases $(0.004 \%)$ the minimum requirement (2 AED failures) was not met. The number of previous AED failures based on EP categorization was lower than that determined by the investigators. As a result of this reclassification, 203 patients (19\% of the entire cohort) were determined by the EP as having failed fewer than 2 AEDs and, as a result, were not considered by the EP as being drug resistant per ILAE criteria and were thus recategorized as "undefined."

Disagreement between the EP and investigators in classifying drug resistance after the prospective trial is influenced by the number of treatment failures categorized at

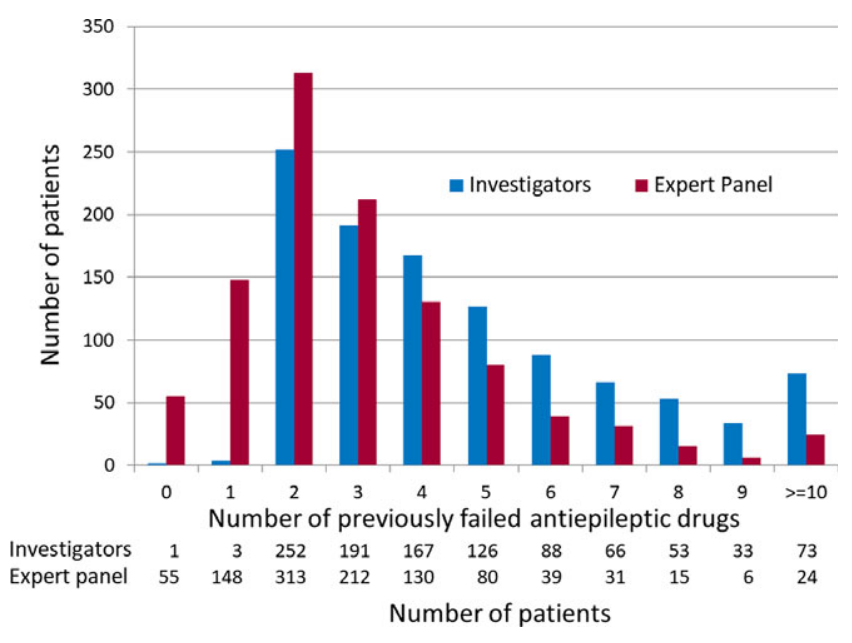

FIGURE 1 Distribution of number of previously failed AEDs at the time of enrollment among the 1053 patients included in the study, as determined by the investigators and by the EP, respectively
TABLE 4 Level of agreement between investigators and $\mathrm{EP}$, and between EP members, in categorizing the outcome of 5725 individual AED trials made up to the time of enrollment (retrospective assessment)

\begin{tabular}{|llllll|}
\hline & $\begin{array}{c}\text { Seizure } \\
\text { freedom }\end{array}$ & $\begin{array}{l}\text { Treatment } \\
\text { failure }\end{array}$ & Undetermined & $\begin{array}{l}\text { No } \\
\text { agreement }\end{array}$ & $\begin{array}{l}\text { Cohen's kappa } \\
\text { coefficient } \\
\text { (95\% CI) }\end{array}$ \\
\hline $\begin{array}{c}\text { Agreement between } \\
\text { investigators and EP }\end{array}$ & 54 & 3095 & 755 & 1821 & $0.50(0.48-0.52)$ \\
\hline $\begin{array}{c}\text { Agreement between } \\
\text { experts at initial } \\
\text { evaluation }\end{array}$ & 51 & 3073 & 2125 & 476 & $0.83(0.81-0.84)$ \\
\hline $\begin{array}{c}\text { Agreement between } \\
\text { experts after attempt } \\
\text { to resolve }\end{array}$ & 51 & 3150 & 2287 & & \\
discrepancies & & & & & \\
\hline
\end{tabular}


enrollment, and therefore will not be reported in detail. Briefly, seizure freedom status was achieved at the end of the study in 92 patients $(8.7 \%)$ as determined by investigators, and in 84 patients $(8 \%)$ as determined by the EP. Of interesting, of the 92 patients classified as seizure-free by the investigators, 19 had been reclassified by the EP as having "undefined" drug responsiveness at enrollment.

\section{4 | DISCUSSION}

Before the present study, 2 separate investigations evaluated interrater reliability in applying the ILAE definition of drugresistant epilepsy. ${ }^{2,10}$ Both studies included a relatively small number of patients $(\leq 150)$ with any type of epilepsy and with different AED responsiveness, and they were based on assessment of medical records by 2 independent evaluators. In the pilot study by Hao et al, ${ }^{10}$ interrater agreement in categorizing drug-responsiveness status (level 2) was excellent $(k=0.91)$, whereas in the study by Tellez-Zenteno et $\mathrm{al}^{2}{ }^{2}$ the level of agreement was inferior $(k=0.77)$, but still satisfactory and better than that observed for other previously used definitions of drug resistance.

The present investigation differed from previously conducted studies in several ways: (a) it included a much larger cohort of consecutively enrolled patients; (b) it focused on adults with focal epilepsy diagnosed by investigators as drug resistant; (c) it included not only a retrospective assessment based on medical records, but also a prospective assessment of the outcome of a therapeutic intervention applied at enrollment, with a prolonged (up to 34-months) duration of follow-up; and (d) interrater agreement was not based on assessment by 2 evaluators but on a comparison between categorizations made by individual investigators at the 43 enrolling centers and by an external $\mathrm{EP}$, as well as between rotating members of EP pairs.

At enrollment, the overall agreement between investigators and the EP in classifying epilepsy as drug resistant was $80 \%$, which is relatively good and close to the level of agreement reported by Tellez-Zenteno et $\mathrm{al}^{2}$ in a more heterogeneous patient group. However, agreement in the level 1 categorization was lower, with a disagreement of $\sim 30 \%$ for the prospective assessment and $\sim 32 \%$ for the retrospective assessment.

Our findings have several practical implications. First, they demonstrate that interrater reliability in assessing treatment outcomes ranges from moderate to almost perfect, being highly dependent on the judgment of individual physicians. Specifically, the level of agreement between different EP members in the blinded assessment of both retrospective $(k=0.83)$ and prospective $(k=0.84)$ AED trials was much greater than the level of agreement between the EP and individual investigators $(k=0.57$ for prospective drug trials; $k=0.50$ for retrospective trials). This observation seems to reflect a more conservative approach of EP members in categorizing the outcome of a therapeutic intervention, both in the retrospective and prospective phases of the study. In fact, in the large majority of cases, disagreement consisted of the EP categorizing as "undetermined" outcomes that had been deemed to be "treatment failures" by the enrolling physicians. The primary causes of disagreement were the EP's determination that data were not sufficiently informative or that the maximal dose achieved was insufficient to consider the trial as adequate. It should be emphasized that application of ILAE criteria is dependent on subjective interpretation of clinical data and, therefore, some degree of individual variability in interpretation is expected. Our findings suggest that a critical determinant of disagreement in interpretation, not only between investigators and the EP, but also between EP members, is the dosage of a drug that should be tried before a treatment can be considered to have failed. The ILAE consensus paper states that "for adults, reference may be made to the WHO's defined daily dose," and that "there should be a documented attempt to titrate the dose to a target clinically effective dose range," but it does not go as far as recommending that, in patients with persisting seizures, an attempt should be made to reach the highest tolerated dose. ${ }^{3}$ Inevitably, physicians' attitudes toward increasing dosage beyond certain limits differ, and there are also inconsistencies in the way clinicians use serum AED levels as a guide to dosage adjustments. Finally, individual patient characteristics and patients' attitudes may affect a prescriber's decision not to increase dosage beyond a certain limit. At least to some extent, these considerations may explain the apparent discrepancies between investigators and the EP in determining which dosages could be regarded as sufficient to categorize an outcome as a treatment failure.

A second important message from our study is that an appreciable number of patients considered to be drug resistant in general clinical practice do not meet the criteria for drug resistance after critical reevaluation of available information. In fact, 1 of 5 patients enrolled by investigators with a diagnosis of drug resistance were not deemed to meet criteria for drug resistance by the EP. Despite the subjective limitations of categorizing treatment outcomes, these data suggest that drug resistance may be overdiagnosed in clinical practice. Similar findings have been reported by other investigators, suggesting that the condition known as "pseudo-pharmacoresistance" is more common than generally recognized. ${ }^{1,11}$ In a study from Spain, only 13 of 40 consecutive patients admitted to an epilepsy unit for presurgical evaluation met the criteria for drug resistance, and $70 \%$ of AED trials previously applied in those patients were considered uninformative. ${ }^{12}$ The authors concluded that adequate information on the implications of the ILAE definition "should be spread among general neurologists 
for earlier and more complete referrals." Before enrollment in our study, all enrolling physicians underwent a training session on the correct application of the definition, but this did not prevent a probable suboptimal categorization of many therapeutic outcomes. Overall, these data suggest that greater efforts should be directed at training physicians on how to critically interpret the outcome of therapeutic interventions. Our findings also suggest that future studies specifically aimed at evaluating different aspects of drug resistance may benefit from including an independent external panel in order to validate, or at least confirm, the diagnosis made by enrolling physicians.

A third message emerging from our results relates to the outcome of the prospective evaluation. A detailed analysis of responses to the newly administered AEDs in relation to the number of previous treatment failures and other individual characteristics is described in a sister publication. ${ }^{8}$ However, a comment is required on the fact that about $8 \%$ of patients confirmed by the EP to be drug resistant at enrollment achieved seizure freedom on a newly administered treatment. This finding supports the validity of the ILAE definition, in that it confirms that for patients fulfilling the criteria for drug resistance "the probability of achieving seizure freedom with subsequent AED treatments is modest." ${ }^{3}$ These data also reinforce the concept that drug resistance is not synonymous with medical intractability, and that some patients can respond to treatment even after failing several AEDs. ${ }^{3}$ In this respect, it should be stressed that our patients had tried more than 5 AEDs in the past and that they represent a subpopulation of more difficult-to-treat patients. Moreover, only one new AED treatment was tried in the intervention, and a few other patients could respond to additional drug trials. To what extent seizure freedom in these patients can be maintained indefinitely is uncertain, because many types of epilepsy seem to follow a relapse-remitting pattern. ${ }^{13}$ The relapsing nature of many types of epilepsy is confirmed by our observation that an appreciable proportion of patients considered drug resistant at enrollment had experienced periods of seizure freedom in the past.

Our study has limitations. First, drug resistance was diagnosed at enrollment by assessing retrospectively outcomes in patients with longstanding epilepsy who had tried on average 5.6 medications, and diagnostic accuracy would be expected to be greater in patients followed up prospectively since diagnosis. Second, inclusion criteria were limited to patients who were considered drug resistant, and only those were reassessed. It is likely that there were other patients, initially not considered drug resistant, that on a formal reassessment as in this study would meet criteria for drug resistance. Third, the study was conducted at secondary and tertiary referral centers, and all participating investigators were neurologists experienced in the management of epilepsy. Therefore, our results cannot be extrapolated to other settings and particularly to the primary care setting where variability in clinical management and in the application of the ILAE definition may be greater. Finally, our study only enrolled adults with focal epilepsy, which ensured assessment of a relatively homogenous population but also prevented inclusion of certain epilepsy syndromes of infancy and childhood, where pseudodrug resistance due to inappropriate AED choice is more likely to occur. ${ }^{14}$

\section{ACKNOWLEDGMENTS}

The authors thank the patients and their caregivers in addition to the investigators and their teams who contributed to this study.

\section{DISCLOSURE OF CONFLICTS OF INTEREST}

This study was sponsored by UCB Pharma, which was involved in the design and conduct of the study, and collection, management, and analysis of the data. The article was written entirely by the authors without external assistance. The authors received no remuneration for the preparation of the manuscript. UCB Pharma was offered a courtesy review of the manuscript, the comments of which were at the authors' discretion to address. GZ has received speaker's or consultancy fees from Eisai, Jansen-Cilag, Sanofi-Aventis, and UCB Pharma. MM has received consultancy fees from UCB Pharma, Eisai, Bial, and Elsevier, and has intellectual property rights with Springer. BF is an employee of UCB Pharma SpA, Italy. AI has received grants for clinical trials and consultancy fees from Bayer, UCB, Novartis, Eisai, FB Health, and Ecupharma. ALN has received speaker's or consultancy fees from Eisai, Mylan, Sanofi-Aventis, and UCB Pharma. MS has received fees for advisory boards and invited lectures from UCB, Eisai, and Sandoz. EP received speaker and/consultancy fees from Eisai, Biopharm Solutions, GW Pharma, LivaNova, Mylan, Sanofi, Sandoz, Shire, Sun Pharma, Takeda, and UCB Pharma. DC, ME, ATG, PT and LZ, declare no conflicts of interest. We confirm that we have read the Journal's position on issues involved in ethical publication and affirm that this report is consistent with those guidelines.

\section{ORCID}

Marco Mula (D) https://orcid.org/0000-0002-9415-3395

Domenico Consoli (iD https://orcid.org/0000-0002-20505538

Maurizio Elia (iD https://orcid.org/0000-0002-0414-1359 Stefano Meletti (iD) https://orcid.org/0000-0003-0334-539X 


\section{REFERENCES}

1. Perucca E. Pharmacoresistance in epilepsy. How should it be defined? CNS Drugs. 1998;10:171-9.

2. Téllez-Zenteno JF, Hernández-Ronquillo L, Buckley S, et al. A validation of the new definition of drug-resistant epilepsy by the International League Against Epilepsy. Epilepsia. 2014;55:829-34.

3. Kwan P, Arzimanoglou A, Berg AT, et al. Definition of drug resistant epilepsy: consensus proposal by the ad hoc Task Force of the ILAE Commission on Therapeutic Strategies. Epilepsia. 2010;51:1069-77.

4. Kwan P, Brodie MJ. Definition of refractory epilepsy: defining the indefinable? Lancet Neurol. 2010;9:27-9.

5. Wiebe S. Definition of drug-resistant epilepsy: is it evidence based? Epilepsia. 2013;54(Suppl 2):9-12.

6. Ramos-Lizana J, Rodriguez-Lucenilla MI, Aguilera-López P, et al. A study of drug-resistant childhood epilepsy testing the new ILAE criteria. Seizure. 2012;21:266-72.

7. Kong ST, Ho CS, Ho PC, et al. Prevalence of drug resistant epilepsy in adults with epilepsy attending a neurology clinic of a tertiary referral hospital in Singapore. Epilepsy Res. 2014;108:1253-62.

8. Mula M, Zaccara G, Galimberi CA, et al. Outcome of treatment changes in adults with drug resistant epilepsy (in preparation).

9. Landis R, Koch GG. The measurement of observer agreement for categorical data. Biometrics. 1977;33:159-74.

10. Hao XT, Wong IS, Kwan P. Inter-rater reliability of the international consensus definition of drug-resistant epilepsy: a pilot study. Epilepsy Behav. 2011;22:388-90.

11. Bajacek M, Hovorka J, Nezadal T, et al. Is pseudo-intractability in population of patients with epilepsy still alive in the 21st century? Audit of 100 seizure-free patients, referred with the diagnosis of pharmacoresistant epilepsy. Neuro Endocrinol Lett. 2010;31:818-22.

12. Carreño M, Aparicio J, Sierra A, et al. Are patients referred for presurgical evaluation drug resistant according to the new consensus definition? A study in a tertiary center. Epilepsy Res. 2012;98:277-80.

13. Brodie MJ, Barry SJ, Bamagous GA, et al. Patterns of treatment response in newly diagnosed epilepsy. Neurology. 2012;78:154855 .

14. Gayatri NA, Livingston JH. Aggravation of epilepsy by anti-epileptic drugs. Dev Med Child Neurol. 2006;48:394-8.

How to cite this article: Zaccara G, Mula M, Ferrò $\mathrm{B}$, et al. Do neurologists agree in diagnosing drug resistance in adults with focal epilepsy? Epilepsia. 2019;60:175-183. https://doi.org/10.1111/epi.14622

\section{APPENDIX}

\section{LIST OF STUDY INVESTIGATORS EXPERT PANEL MEMBERS}

Emilio Perucca (Pavia), Chair

Maria Paola Canevini (Milan)

Ornella Daniele (Palermo)

Maurizio Elia (Troina)
Carlo Andrea Galimberti (Pavia)

Anna Teresa Giallonardo (Roma)

Alfonso Iudice (Pisa)

Angela La Neve (Bari)

Oriano Mecarelli (Roma)

Roberto Michelucci (Bologna)

Marco Mula (London, UK)

Andrea Romigi (Roma)

Luigi Maria Specchio (Foggia)

Salvatore Striano (Napoli)

Paolo Tinuper (Bologna)

Gaetano Zaccara (Firenze)

\section{STATISTICAL ANALYSIS}

Antonio Colantoni, Hippocrates Research Srl, Genoa, Italy

\section{CLINICAL STUDY INVESTIGATORS}

Carlo Andrea Galimberti, Cristina Valisi (Pavia); Francesco Paladin, Filippo Dainese (Venezia); Roberto Eleopra, Christian Lettieri, Giada Pauletto (Udine); Tiziano Zanoni, Monica Ferlisi (Verona); Francesco Pierini, Federica Ranzato (Vicenza); Teresa Anna Cantisani, Chiara Piersanti, Federica Nardini, Michela Cecconi (Perugia); Nicoletta Foschi, Chiara Fiori, Cristina Petrelli (Ancona); Raffaele Rocchi, Lorenzo Celli, Daniela Marino (Siena); Paolo Aloisi (L'Aquila); Alfonso Iudice, Emanuele Bartolini (Pisa); Marco Paganini, Anna Maria Romoli, Francesca Pizzo (Firenze); Gaetano Zaccara, Luciana Tramacere (Firenze); Angela La Neve, Teresa Francavilla, Marianna Ladogana (Bari); Nicola Paciello (Potenza); Giovanni Boero (Taranto); Dolores Mascia (Pozzilli); Luigi Maria Specchio, Maria Grazia Pascarella (Foggia); Anna Teresa Giallonardo, Jiinane Fattouch, Sara Casciato (Roma); Oriano Mecarelli, Alessia Zarabla (Roma); Michele Feleppa, Maria Rosaria D'Argenio (Benevento); Francesco Habetswallner, Bernardo De Martino, Amelia Cutolo (Napoli); Maria Grazia Marciani, Andrea Romigi, Maria Albanese (Roma); Salvatore Striano, Marta Ianniciello, Marianna De Vito, Luigi Del Gaudio (Napoli); Silvana Franceschetti, Davide Sebastiano Rossi (Milano); Flavio Villani, Giuseppe Didato (Milano); Fabio Minicucci, Emanuela Leopizzi (Milano); Stefano Meletti, Laura Mirandola, Giada Giovannini (Modena); Roberto Michelucci, Patrizia Riguzzi (Bologna); Paolo Tinuper Barbara Mostacci, Marcella Broli (Bologna); Maria Paola Canevini, Giulia Scornavacca (Milano); Gianpietro Sechi, Silia Fenu, Chiara Fois (Sassari); Francesco Marrosu, Gianni Orofino (Cagliari); Vito Sofia, Irene Pappalardo, Chiara Sueri, Loretta Giuliano (Catania); Ornella Daniele, Leila Zummo (Palermo); Francesco Pisani, Laura Rosa Pisani, Giuseppe Loddo, Cesare Stilo (Messina); Umberto Aguglia, Cinzia Leonardi, Edoardo Ferlazzo, 
Marina Angela Bellavia (Reggio Calabria); Domenico Consoli, Caterina Gattuso (Vibo Valentia); Maurizio Elia, Maria Grazia Figura (Troina); Gianandrea Ottonello, Daniela Arecco (Genova); Antonio Gambardella, Maria
Pantusa (Catanzaro); Fabrizio Monti, Marco Belluzzo, Marinella Tomaselli (Trieste); Luigi Giuseppe Bongiovanni, Francesco Brigo (Verona); Paola Banfi, Valeria Mariani (Varese). 